\title{
Efecto del sistema silvopastoril con Alnus acuminata en el valor agronómico y nutricional del Pennisetum clandestinum
}

\section{Effect of the silvopastoral system with Alnus acuminate on the agronomic worth and nutritional compsition of Pennisetum clandestinum}

\author{
Beyner Tafur Sanchez
}

\begin{abstract}
RESUMEN
El objetivo de la investigación fue evaluar el efecto del sistema silvopastoril con Alnus acuminata en el valor agronómico y nutricional del Pennisetum clandestinum. Se seleccionó dos sistemas silvopastoril (SSP) de una edad aproximada de 8 años, además un tratamiento repeticiones con medidas iguales, se empezó a recolectar muestras de manera aleatoria con ayuda de un cuadrante para su posterior evaluación en el laboratorio a los 30,45 testigo, las cuales fueron divididas en 4 y 60 días. Las variables fueron analizadas usando un diseño completamente al azar, mediante la prueba de comparaciones múltiples (Tukey). Los valores de proteína cruda alos 45 días mostraron diferencias significativas donde la proteína cruda bajo los SSP mostró valores de $17.77 \%$ en el primer SSP y $17.01 \%$ en el segundo SSP, estos superiores al CA (15.04\%). Lo contrario sucedió con la FDA, FDN y ELN, donde la FDA bajo los SSP obtuvo valores de $27.01 \%$ y $28.73 \%$ y el CA $29.14 \%$ de igual manera los valores de FDN fueron de $56.68 \%, 54.46 \%$ y el CA $60.65 \%$, por consiguiente, el ELN obtuvo valores de $36.46 \%, 37.19 \%$ respecto al CA que generó $38.92 \%$ respectivamente, por lo tanto, los SSP tiene un efecto positivo sobre la calidad del forraje ayudando así a una mejor digestibilidad.
\end{abstract}

Palabras clave: Sistema silvopastoril, valor agronómico

\begin{abstract}
The objective of the research was to evaluate the effect of the silvopastoral system with Alnus acuminata on the agronomic worth and nutritional of Pennisetum clandestinum, two SSP of an approximate age of eight years were selected, in addition to a control treatment, which were divided into 4 repetitions with equal measures, samples were randomly collected with the help of a quadrant for subsequent evaluation in the laboratory at thirty, forty five and sixty days. Variables were analyzed using a completely randomized design, using the multiple comparison test (Tukey). The crude protein values at forty five days of cut showed significantm differences where the crude protein under the SSP showed values of $17.77 \%$ in the first SSP, $17.01 \%$ in the second SSP, these higher than the CA (15.04\%). The opposite happened with the FDA, FDN and ELN, where the FDA under the SSP obtained values of $27.01 \%$ and $28.73 \%$ and the CA $29.14 \%$ in the same way the values of FDN they were $56.68 \%$, $54.46 \%$ and the CA $60.65 \%$, therefore, the ELN obtained values of $36.46 \%, 37.19 \%$ with respect to the CA that generated $38.92 \%$ respectively, therefore the SSP has an effect positive on forage quality thus helping better digestibility.
\end{abstract}

Keywords: Silvopastoral system, agronomic value 


\section{INTRODUCCIÓN}

Hoy en día la ganadería exige mejorar la productividad por lo que los ganaderos buscan opciones para reducir costos y tener una mayor eficacia de rentabilidad (Demara, 2015).

En los últimos años los bosques que se han deforestado en la amazonia, han sido establecidos con pastizales para la actividad ganaderas. Estas praderas, utilizadas sin técnicas de manejo adecuadas, lo cual ocasiona efectos negativos, produciendo así pérdida de nutrientes, por consiguiente, la mejor alternativa de solución es el manejo adecuado de los suelos, mediante los sistemas silvopastoriles (SSP). Esta alternativa permite disminuir los procesos de degradación de los suelos, mediante la incorporación de la materia orgánica que proviene de los restos vegetales. Además, estos sistemas permiten el mejoramiento de las propiedades físicas, químicas y biológicas del suelo.

Los sistemas silvopastoriles con especies arbóreas como el aliso en asociación con cualquier especie forrajera están siendo consideradas como nuevas alternativas de explotación agrícola ya que son biológica, económica y ecológicamente más sustentable que los sistemas tradicionales (Alegre, 2019).

Los pastos y los forrajes son la principal fuente de alimento para los rumiantes y representan el mayor volumen de la dieta, ya que son más baratos, tienen una gran capacidad de producción y crecen fácilmente (Olafadehan, 2019).

La producción ganadera depende, en gran medida, de los factores climáticos y meteorológicos; por ello, la transición anual de las condiciones climáticas, fundamentalmente en cuanto a la temperatura, la humedad relativa y las precipitaciones, genera la correspondiente variabilidad en la producción y el valor nutritivo de los forrajes. En tal sentido, la ganadería basada en el pastoreo de gramíneas en monocultivo enfrenta grandes desafíos, especialmente en la época de sequía, durante la cual disminuyen de manera drástica la disponibilidad y la calidad de los forrajes (Cardona et al., 2014). Por otra parte, la determinación de la capacidad de producción actual de los pastos, junto a la presión en relación con el uso de la tierra para la producción ganadera, se ha convertido en uno de los principales desafíos para desarrollar la planificación integrada y la toma de decisiones en los sistemas de pastoreo, ello explica la necesidad de adoptar sistemas sostenibles de producción ganadera que aprovechen las ventajas del manejo integrado. Por tanto, la conversión ambiental basada en el silvopastoreo constituye una alternativa promisoria para enfrentar estos problemas ya que los sistemas con árboles son capaces de conservar la biodiversidad (Schindler et al., 2016), aportar servicios ambientales a los ecosistemas, incrementar tanto cualitativa como cuantitativamente la oferta de forraje al ganado, así como minimizar el desbalance en la producción de alimentos que caracteriza a los sistemas sin árboles (Restrepo et al., 2016). La composición química y la morfología de los forrajes determinan la palatabilidad y el valor nutricional para el ganado, por lo cual influyen en la cantidad de alimento que se consume, la eficiencia de la rumia, la tasa de ganancia de peso, el volumen y la calidad de la leche que se produce, y en el éxito reproductivo (Herrero, 2019).

Por otro lado, el silvopastoreo con respecto a potreros con monocultivo de gramíneas han sido identificadas en diversos estudios científicos, reconocidas por los productores, donde los monocultivos presentan baja tolerancia a la sequía por lo que en época seca disminuye la calidad de su forraje y la producción de materia seca, afectando la productividad animal; en contraste, con los SSP se evidencia mayor estabilidad en la producción y disponibilidad de forraje $\mathrm{y}$ nutrientes durante todo el año (Buitrago et al.,2018).

El objetivo de esta investigación fue evaluar el efecto del sistema silvopastoril con Alnus acuminata en el valor agronómico y nutricional del Pennisetum clandestinum, se seleccionó dos SSP de una edad aproximada de 8 años, además un tratamiento testigo, las cuales fueron divididas en 4 repeticiones con medidas iguales, se empezó a recolectar muestras de manera aleatoria con ayuda de un cuadrante para su posterior evaluación en el laboratorio a los 30,45 y 60 días. Las variables fueron analizadas usando un diseño completamente al azar, mediante la prueba de comparaciones múltiples (Tukey). Los valores de proteína cruda a los 45 días mostraron diferencias significativas donde la proteína cruda bajo los SSP mostró valores de $17.77 \%$ en el primer SSP y $17.01 \%$ en el segundo SSP, estos superiores al CA (15.04\%). En conclusión, los SSP tiene un efecto positivo sobre la calidad del forraje ayudando así a una mejor digestibilidad.

\section{MATERIALES Y METODOS}

\subsection{Ubicación}

El presente estudio se desarrolló en el distrito de Florida-Pomacochas, provincia Bongará (E.E. Pomacochas de la Universidad Nacional Toribio Rodríguez de Mendoza); departamento de Amazonas, a una altitud de $2232 \mathrm{msnm}$.

\subsection{Selección de los sistemas silvopastoriles (SSP)}

Se realizó una selección de parcelas que tuvieron como componente forrajero Pennisetum clandestinum y como parte del componente arbóreo a las especies nativas Alnus acuminata (aliso) de una edad aproximada de 8 años. La muestra estuvo conformada por dos parcelas con sistema 
silvopastoril (Alnus acuminata + Pennisetum clandestinum) la primera con un diseño rectangular de 3 x 5 (3 metros entre árboles y 5 metros entre surcos). El segundo con un diseño rectangular de $3 \mathrm{x}$ 10 (3 metros entre árboles y 10 metros entre surcos). Adicionalmente un tratamiento testigo (campo abierto). Cada tratamiento con 4 repeticiones respectivamente, haciendo un total de 12 parcelas; donde cada parcela constaba un área de 40 metros cuadrados ( $5 \mathrm{~m} \times 8 \mathrm{~m})$.

\subsection{Determinación del valor agronómico del forraje}

\section{a) Rendimiento de forraje verde (biomasa)}

La cuantificación del rendimiento de forraje verde (biomasa) se realizó mediante el método del cuadrante de 0,5 m x 0,5 m2 (Rojas et al., 2014), los cuales se lanzaron a una distancia aproximadamente de $0.5 \mathrm{~m}$ del tronco de los aboles para posteriormente cortar el componente forrajero con ayuda de un hoz de cada unidad experimental a una edad de 30, 45 y 60 días respectivamente, posterior a ello se tomaron 4 submuestras por cada parcela simulando el consumo del animal a una altura mínima de $3 \mathrm{~cm}$ del suelo, para luego pesarlas en una balanza digital y así determinar el peso total por metro cuadrado y proyectarlos a toneladas por hectárea (ton./ha), según la metodología descrita por (Lascano \& Pizarro, 1984).

\subsection{Determinación del valor nutricional del forraje.}

\section{a) Proteína cruda (PT)}

Se determinó mediante el método de kjelhal automático, el cual comprende tres fases: digestión, destilación y titulación obteniendo como resultado final la cantidad de nitrógeno total (método 928.08) según la AOAC.

\section{RESULTADOS}

\subsection{Análisis de composición bromatológica del Pennisetum clandestinum.}

A los 45 días de corte valores promedios de proteína cruda (PC) mostraron diferencias significativas al $(\mathrm{p}<0.05)($ tabla 1$)$.

Tabla 1: Comparación de media de $\mathrm{PC}$ a los 45 días de corte.

\begin{tabular}{cc}
\hline & Composición bromatológica \\
\cline { 2 - 2 } Tratamiento & $15.04^{\mathrm{b}}$ \\
CA & $17.77^{\mathrm{a}}$ \\
SSP(3X5) & $17.01^{\mathrm{ab}}$ \\
SSP(3X10)
\end{tabular}

Figura 2:Composición de PC del forraje a los 45 días de corte

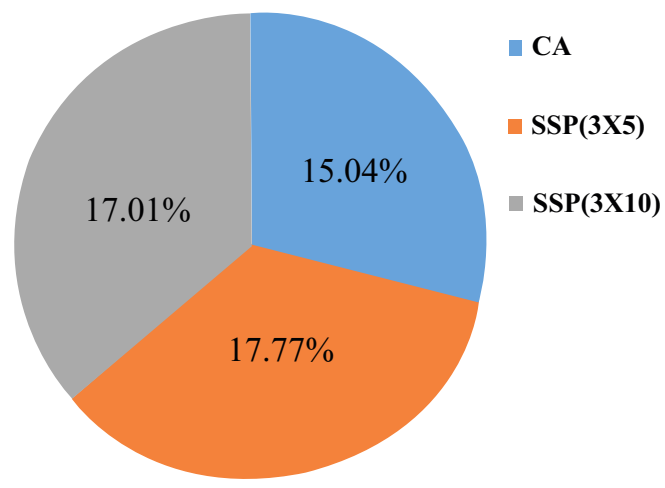

En la figura 1, se muestra valores de PC bajo los SSP superiores con $17.77 \%, 17.01 \%$ en relación al CA cuyo valor obtenido fue de $15.04 \%$.

\subsection{Análisis del valor agronómico del Pennisetum clandestinum.}

En los sistemas silvopastoriles y campo abierto (CA) a los 45 días de corte se evidenció diferencias significativas $(\mathrm{p}<0.05)$ para biomasa (Tabla 2$)$.

Tabla 2:Comparación de media de biomasa a los 45 días de corte.

\begin{tabular}{cc}
\hline & Valor agronómico 45 días de corte \\
\cline { 2 - 2 } Tratamiento & Biomasa $\left(\mathbf{K g} / \mathbf{m}^{2}\right)$ \\
CA & $2.785^{\mathrm{b}}$ \\
SSP(3X5) & $3.465^{\mathrm{b}}$ \\
SSP(3X10) & $3.315^{\mathrm{ab}}$ \\
\hline
\end{tabular}

Figura 1:Biomasa del forraje a los 45 días de corte.

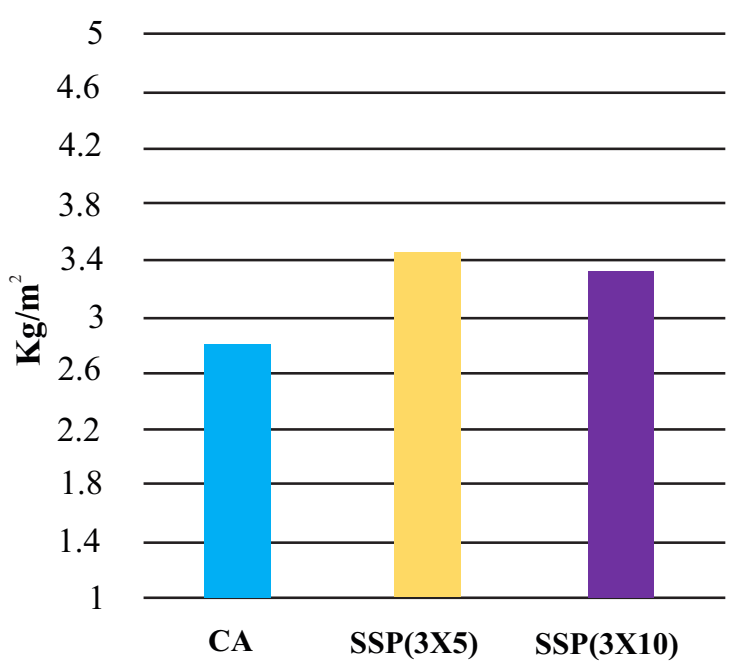


En la figura 2, se muestra valores de biomasa en el $\mathrm{SSP}$ que fueron superiores en $0.680 \mathrm{~kg} / \mathrm{m} 2$ y 0.530 $\mathrm{kg} / \mathrm{m} 2$ respecto al CA.

Valores promedios de biomasa del CF bajo los sistemas silvopastoriles analizados mostraron diferencias significativas al $(\mathrm{p}<0.05)$ a los 60 días de corte (Tabla 3).

Tabla 3: Comparación de media de biomasa a los 60 días de corte.

\begin{tabular}{cc}
\hline & Valor agronómico 60 días de corte \\
\cline { 2 - 2 } Tratamiento & Biomasa $\left(\mathbf{K g} / \mathbf{m}^{2}\right)$ \\
CA & $3.336^{\mathrm{b}}$ \\
SSP(3X5) & $3.985^{\mathrm{ab}}$ \\
SSP(3X10) & $4.178^{\mathrm{a}}$
\end{tabular}

Tabla 3: Comparación de media de biomasa a los 60 días de corte.

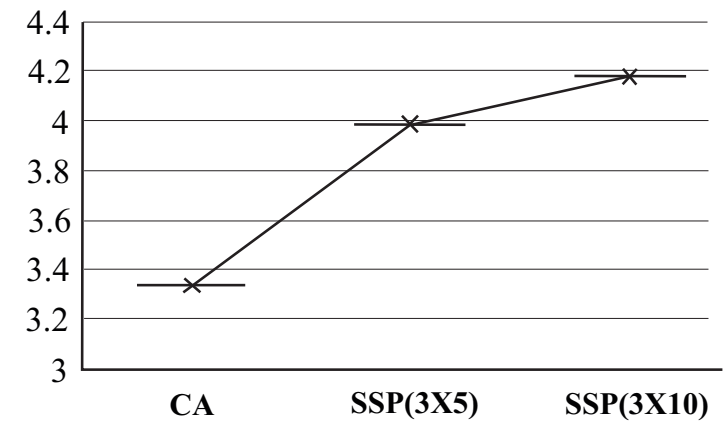

En la figura 3, se observa que los valores de biomasa bajo los SSP son mayores en $0.649 \mathrm{~kg} / \mathrm{m} 2,0.842$ $\mathrm{kg} / \mathrm{m} 2$ respecto al $\mathrm{CA}$, de igual manera el rendimiento de materia seca bajo SSP son más altos con valores de $0.751 \mathrm{~kg} / \mathrm{m} 2$ en el SSP $(3 \times 5), 0.795$ $\mathrm{kg} / \mathrm{m} 2$ en el SSP $(3 \times 10)$ y $0.559 \mathrm{~kg} / \mathrm{m} 2$ en el CA.

\section{DISCUSIÓN}

4.1. Análisis de composición bromatológica del Pennisetum clandestinum en un sistema silvopastoril con Alnus acuminata.

A los 30 días de corte la evaluación de proteina cruda (PC) del CF mostro niveles mas altos generando así valores de $16.51 \%$ (CA), $18.05 \%$ el SSP (3x5) y $17.25 \%$ el SSP $(3 \times 10)$, estos valores superiores a los reportados por Silva, (2018) donde encontro valores de $15.13 \%$ de PC bajo un SSP con $A$. acuminata y $7.73 \%$ en CA, sin embargo alos 45 dias de corte se mostraron diferencias significativas generando así cada tratamiento valores de 15.04\% (CA), $17.77 \%$ el SSP $(3 \times 5)$ y $17.01 \%$ el SSP $(3 \times 10)$, estos valores superiores a los encontrados por Espinoza (2018), que evaluó productividad y calidad nutritiva de pastos en sistemas silvopastoriles con diferentes especies arbóreas dentro de ellas con
Alnus acuminata en asociación con diferentes especies forrajeras como el Pennisetum clandestinum en la microcuenca de molinopampa, donde el CF mostró valores de proteína cruda de $14.17 \%$ en campo abierto y $16.50 \%$ bajo SSP con Alnus acuminata.

También (Chamorro \& Rey, 2010), reporta mejores niveles de proteína en el forraje que está asociado con Alnus acuminata $17.1 \%$, para el primer SSP, mientras que para el segundo sistema fue $16.1 \%$, estos reportes superiores al monocultivo de Pennisetuim clandestinum (15.6\%).

Aquí se puede identificar que el CF existente en campo abierto son los que aportan menor contenido de proteína, mientras que los SSP generan una mayor concentración de proteína en el CF, esto puede estar asociado al efecto de retención de humedad y fijación de nitrógeno de $A$. acuminata.

Al parecer el efecto complementario de esta especie arbórea en praderas de $P$. clandestinum se refleja en una mayor disponibilidad y calidad de forraje. En este trabajo de investigación el Pennisetum clandestinum reporto valores de PC superiores a lo normal esto debido a que fue sometido a un abonamiento con gallinaza a una dosis de $2.5 \mathrm{~kg} / \mathrm{m} 2$ y para corroborar dicho resultado se recolectó muestras de parcelas sin abonamiento pudiendo constatar valores inferiores con reportes de $16.62 \%$ bajo el SSP y 15.32 en CA mientras que en esta investigación se evidenció valores de $18.05 \%$ como máximo bajo el SSP y $16.51 \%$ en CA, valores superiores reportó (Correa C, 2012), en su trabajo experimental denominado "Valor nutricional del Pennisetum clandestinum para la producción de leche" cuyos reportes de PC fue de $20.5 \%$, el contenido de proteína en este pasto se debió a que normalmente es sometido a intensos programas de fertilización nitrogenada y es pastoreado a edades más cortas que en el pasado.

4.2.Análisis del valor agronómico del Pennisetum clandestinum en un sistema silvopastoril con Alnus acuminata.

A los 45 días de corte la variable biomasa del CF presentó diferencias en sus valores, donde la biomasa calculada bajo los SSP $(3 \times 5)$ y $(3 \times 10)$ superaron en $0.680 \mathrm{~kg} / \mathrm{m} 2$ y $0.530 \mathrm{~kg} / \mathrm{m} 2$ al tratamiento testigo (CA).

A los 60 días de corte se evidenció diferencias en la variable biomasa. La biomasa calculada en los SSP con diseño rectangular $(3 \times 5)$ y $(3 \times 10)$ fueron relativamente superiores en $0.649 \mathrm{~kg} / \mathrm{m} 2$ y 0.842 $\mathrm{kg} / \mathrm{m} 2$ al tratamiento testigo (CA). El componente forrajero también presenta diferencia significativa $(\mathrm{p}<0.05)$ a los 60 días de corte en cuanto a biomasa, donde sus valores en CA para biomasa fue 3.336 $\mathrm{kg} / \mathrm{m} 2,3.985 \mathrm{~kg} / \mathrm{m} 2$ en SSP $(3 \times 5)$ y $4.178 \mathrm{~kg} / \mathrm{m} 2$ en el SSP $(3 \times 10)$ estos valores inferiores a los reportados 
por Insuasty (2011), en su trabajo de investigación donde encontró valores de $1.391 \mathrm{~kg} / \mathrm{m} 2$ en CA y $1.964 \mathrm{~kg} / \mathrm{m} 2$ bajo el SSP con A. acuminata. Esta diferencia de valores quiza se deba a la calidad del suelo donde se desarrollo la investigación asi como también la edad de corte del CF y densidad de siembra de la especie arborea instalada.

\section{CONCLUSIONES}

El pasto kikuyo (Pennisetum clandestinum) en el $\mathrm{SSP}$, permaneció en mejores condiciones por la presencia del aliso (Alnus acuminata); comparativamente con el campo abierto.

Las características agronómicas evaluadas tales como biomasa y producción de MS en el SSP fueron mejores que las presentadas en el tratamiento testigo (campo abierto) a los 60 días de corte, donde el rendimiento de biomasa en los SSP fueron superiores en $0.649 \mathrm{~kg} / \mathrm{m} 2$ y $0.842 \mathrm{~kg} / \mathrm{m} 2$ al tratamiento testigo (CA).

Los rendimientos obtenidos para MS del CF bajo los SSP se incrementaron en $0.192 \mathrm{~kg} / \mathrm{m} 2$ y 0.236 $\mathrm{kg} / \mathrm{m} 2$ respectivamente. A. acuminata influyo directamente en la calidad bromatológica del $P$. clandestinum, quizás debido a los efectos de retención de humedad, generando así mayor humedad al CF a los 30 días de corte. Estos valores pueden estar relacionados con la fijación biológica del nitrógeno, así como también por la sombra, permitiendo altos contenidos de nutrientes en el CF.

En esta investigación mayores concentraciones de PC se obtuvo a los 30 días de corte donde los valores de los SSP $(3 \times 5)$ y $(3 \times 10)$ superaron en $1.54 \%$ y $0.74 \%$ al CA, sin embargo, mayores diferencias significativas en los parámetros de la composición nutricional del CF se mostraron a los 45 días de corte donde se evidenció un incremento del $0.46 \%$ y $0.64 \%$ de extracto etéreo en los SSP respecto al CA.

Los SSP con diseño $(3 \times 5)$ y $(3 \times 10)$ registraron los valores más bajos de FDA: $(27.01 \%$; 28.73\%) mientras que el CA registró $29.14 \%$ de igual manera las concentraciones de FDN: $(56.68 \% ; 54.46 \%)$ y el CA $60.65 \%$ por ende las concentraciones de ELN bajo los SSP mostraron $36.46 \%$ y $37.19 \%$ estos valores menores al tratamiento testigo que reportó $38.92 \%$.

\section{REFERENCIAS BIBLIOGRÁFICAS}

Amanda Silva Parra, S. G. (Junio de 2018). IMPACTO DE Alnus acuminata Kunth EN LOSFLUJOS DE $N \quad 2 \quad O \quad Y$ CALIDAD DEL PASTOPennisetum clandestinum Hochst. ex Chiov. Obtenido de http://www.scielo.org.co/scielo. php?script $=$ sci_arttext $\&$ pid $=$ S 0120 07392018000100047

Barreto, D. (2015). DINAMICA DE CRECIMIENTO DE UNA PRADERA Brachiaria brizantha CV TOLEDO EN FUNCIONDE LA S VARIABLES DASOMETRICAS. Obtenido de http://repository.lasalle.edu.co/b itstream/handle/10185/17654/13 062067 \%202015.pdf?sequence $=3 \&$ isAllowed $=\mathrm{y}$

Buitrago-Guillen, M. E., Ospina-Daza, L. A., \&

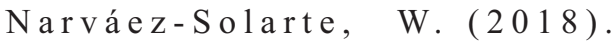
SISTEMAS SILVOPASTORILES: ALTERNATIVA EN LAMITIGACIÓN $Y$ ADAPTACIÓNDE LA PRODUCCIÓN BOVINA AL CAMBIO CLIMÁTICO. Boletin Cientifico Del Centro de Museos, 22(1), 31-42.

Carlos Lascano, E. P. (2015). Evaluacion de pasturas con animales (Issue 1). https://doi.org/10.16309/j.cnki.is sn.10071776.2003.03.004

Correa C, P. R. (diciembre de 2012). Valor nutricional del pasto kikuyo (Pennisetum clandestinum Hoechst Ex Chiov.) para la producción de leche en Colombia (Una revisión): I - Composición química y digestibilidad ruminal y posruminal.

Cuartas Cardona, N. R. (2014). Contribution of intensive silvopastoral systems to animal performance and to adaptation and mitigation of climate change.

Chamorro Viveros, D., \& Rey O., A. M. (Abril de 2010). Los sistemas silvopastoriles como estrategiade ganaderia ecologica yproductiva.

Escobar Espinoza, L. O. (2018). Productividad y calidad nutritiva de pastos en sistemas silvopastoriles con diferentes especies arboreas en la microcuenca de molinopampa.

Gomez Insuasty, A. S., Silva Parra, A., Jader Salazar, J., \& Andrade Garcia, J. (Octubre de 2011). Producción de materia seca y calidad del pasto kikuyo P. clandestinum en diferentes niveles de fertilización nitrogenada y en 
asocio con aliso alnus acuminata en el trópico alto colombiano. Obtenido de https://ainfo.cnptia.embrapa.br/d igital/bitstream/item/123660/1/p 32-41Doc.-268-Anais.pdf

José Edmundo Apráez Guerrero, F. N. (Septiembre de 2011). Efecto del arreglo silvopastoril aliso (AlnusAcuminata K.) $y$ kikuyo(PennisetumClandestinu $m$ H.) sobre el comportamiento productivo en novillas Holstein.

Julio Alegre, Y. S. (2019). Manejo de suelos con sistemas silvopastoriles en las regiones de Amazonas y San Martin. Chachapoyas.

López-Vigoa, O., Lamela-López, L., SánchezSantana, T., Olivera- Castro, Y., GarcíaLópez, R.,Herrera-Villafranca, M., \& González-Ronquillo, M. (2019). Evaluación del valor nutricional de los forrajes en un sistema silvopastoril. Pastos y Forrajes, 42(1), 57-67.

Milera, M. (2013). Contribución de los sistemas silvopastoriles en la producción y el medio ambiente. Avances En Investigación Agropecuaria, 17(3), 7-24.

Murgueitio Restrepo, E., Barahona Rosales, R., Flores Estrada, M. X., Chará Orozco, J. D., \& Rivera Herrera, J. E. (2016). Es Posible Enfrentar el Cambio Climático y Producir másLechey Carne con Sistemas Silvopastoriles Intensivos. Ceiba, 54(1), 23-30.

Olafadehan, O. A. (2019). Evaluación del valor nutricional de los forrajes en un sistema silvopastoril. Obte $\mathrm{nido} \mathrm{de}$ http://scielo.sld.cu/scielo.php?sc ript $=$ sci_arttext \& pid=S 0864 03942019000100057 